\title{
Effects of mesenteric vein L-alanine infusion on liver metabolism in beef heifers fed on diets differing in forage:concentrate ratio*
}

\author{
BY C. K. REYNOLDS AND H.F. TYRRELL $\dagger$ \\ USDA, Agricultural Research Service, Livestock and Poultry Sciences Institute, \\ Ruminant Nutrition Laboratory, Beltsville, MD 20705, USA
}

(Received 19 June 1990 - Accepted 5 March 1991)

\begin{abstract}
Effects of $24 \mathrm{~h}$ mesenteric vein infusion of $L$-alanine $(75 \mathrm{mmol} / \mathrm{h})$ and diet forage: concentrate ratio on net nutrient metabolism by portal-drained viscera (PDV) and liver were measured in four Hereford-Angus heifers. Heifers were fed on diets containing approximately $750 \mathrm{~g}$ lucerne (Medicago sativa) or $750 \mathrm{~g}$ concentrate/ $\mathrm{kg}$ every $2 \mathrm{~h}$ at similar metabolizable energy intakes in a balanced single reversal design with 6 weeks for diet adaptation. Measurements of blood flow and net metabolism of nutrients were obtained at $\mathbf{3 0}$ min intervals starting immediately before (four measurements) and after (five measurements) beginning, immediately before ending (four measurements) and $1.5 \mathrm{~h}$ after ending (three measurements) alanine infusion. Only values obtained immediately before beginning and ending alanine infusion were compared statistically. Net total splanchnic (PDV plus liver) release of glucose was greater when heifers were fed on the $750 \mathrm{~g}$ concentrate $/ \mathrm{kg} v .750 \mathrm{~g}$ lucerne $/ \mathrm{kg}$ diet due to greater net PDV absorption. Net PDV absorption of ammonia-nitrogen, $\beta$-hydroxybutyrate (BOHB) and lactate, and net liver and total splanchnic release of urea-N (UN) were greater when heifers were fed on the $750 \mathrm{~g}$ lucerne $/ \mathrm{kg} v .750 \mathrm{~g}$ concentrate $/ \mathrm{kg}$ diet. Infusion of alanine increased net liver removal of alanine and $\alpha$-amino- $N$, net liver release of $\mathrm{UN}$ and net total splanchnic release of alanine, $\mathrm{UN}$ and lactate. Infusion of alanine decreased net liver release of $\mathrm{BOHB}$ and removal of lactate. Mesenteric vein infusion of alanine altered the net availability of precursors for liver glucose synthesis, but did not affect net glucose release by liver in these beef heifers.
\end{abstract}

Cattle : Liver: L-Alanine

Although not considered an essential dietary nutrient for non-ruminants, alanine is an important participant in the inter-organ transfer of carbon and nitrogen in both nonruminants and ruminants. Alanine removed by liver is derived predominantly from the portal-drained viscera (PDV) (gastrointestinal tract, pancreas, spleen plus mesenteric and omental fat) in fed sheep and from peripheral tissues (primarily muscle) in fasted sheep (Bergman \& Heitmann, 1978). C from alanine readily exchanges with lactate and glucose pools via pyruvate or intermediates of the tricarboxylic acid (TCA) cycle (Hetenyi et al. 1980). The $\mathrm{N}$ from alanine appears in a number of metabolites including urea, glutamate and glutamine. In sheep, peripheral tissues remove glutamate and release glutamine, and together alanine, glutamate and glutamine account for $7-15 \%$ of liver glucose synthesis (Bergman \& Heitmann, 1978).

In a previous study (Reynolds et al. 1991), net alanine release by PDV of growing

* Mention of a trade name, proprietary product, or specific equipment does not constitute a guarantee or warranty by the US Department of Agriculture and does not imply its approval to the exclusion of other products that may be suitable.

$\dagger$ Present address: Plant and Animal Sciences Staff, Cooperative State Research Service, Aerospace Building, Suite 330, 901 D Street SW, Washington, DC 20250-2200. 
beef heifers fed on two diets differing in forage: concentrate ratio accounted for $27 \%$ of net PDV absorption of $\alpha$-amino-N (AAN). Net PDV release of alanine increased with metabolizable energy (ME) intake but was not affected by diet composition and amount of $\mathrm{N}$ digested. In addition, net liver removal of alanine always matched net PDV release such that, regardless of ME intake, net total splanchnic flux (PDV plus liver) of alanine was near zero and the ratio liver glucose release: liver alanine removal was constant. Therefore, the present study was conducted to determine effects of additional alanine absorption (simulated by mesenteric vein infusion) and diet forage:concentrate ratio on liver metabolism of alanine, glucose, urea-N (UN) and other metabolites in beef heifers.

\section{MATERIALS AND METHODS}

\section{Animals and diets}

Four non-pregnant Hereford-Angus heifers (443 kg body-weight) were used, surgically prepared with chronic blood sampling catheters enabling measurement of visceral nutrient metabolism. Heifers had completed a previous study (Reynolds et al. 1991) for which catheters were surgically implanted at $256 \mathrm{~kg}$ body-weight. Heifers were housed in a tie stall barn under constant temperature $\left(18^{\circ}\right)$, relative humidity $(60 \%)$ and $17 \mathrm{~h}$ light $-7 \mathrm{~h}$ dark cycle, were exercised daily and had constant access to water and trace-mineralized salt (Cargill, Inc., Minneapolis, MN). Heifers were fed on each of two pelleted diets (Table I) containing approximately $(\mathrm{g} / \mathrm{kg}$ ): 750 concentrate, 250 lucerne (Medicago sativa) or 750 lucerne, 250 concentrate, in a balanced single reversal design. At completion of the previous study, heifers had been fed on one of the two diets for at least 10 weeks (two heifers per diet). Heifers continued to receive a relatively low intake of the same diets (70 or $65 \mathrm{~g}$ as $\mathrm{fed} / \mathrm{kg}$ body-weight $\mathrm{t}^{0.75}$ for the $750 \mathrm{~g}$ lucerne $/ \mathrm{kg}$ and $750 \mathrm{~g}$ concentrate $/ \mathrm{kg}$ diets respectively) for a minimum of 4 weeks before initial measurements of net visceral metabolism were obtained for the present study. Diets were then changed and measurements were repeated after a 6-week diet adaptation period. Body-weight was measured on Tuesday and Friday and intakes were adjusted weekly based on average body-weight for the preceding week. Beginning at least $10 \mathrm{~d}$ before measurements of net visceral metabolism, daily rations were given as twelve equal meals delivered every $2 \mathrm{~h}$ to minimize postprandial responses. Diets given for 1 week preceding and during measurements of net visceral metabolism were preweighed from one pellet mill batch and sampled for chemical analysis. Daily intakes averaged $6.15 \mathrm{~kg}$ dry matter (DM), $175 \mathrm{~g} \mathrm{~N}$ and $60.0 \mathrm{MJ} \mathrm{ME}$ for the $750 \mathrm{~g}$ lucerne $/ \mathrm{kg}$ diet and $5.51 \mathrm{~kg} \mathrm{DM}, 149 \mathrm{~g} \mathrm{~N}$ and $66.4 \mathrm{MJ} \mathrm{ME}$ for the $750 \mathrm{~g}$ concentrate $/ \mathrm{kg}$ diet.

\section{Alanine infusion, blood sampling and analyses}

Beginning $0.5 \mathrm{~h}$ before feeding at 07.30 hours, nine simultaneous $20 \mathrm{ml}$ blood sample sets were obtained from the aorta and hepatic and portal veins at 30 min intervals for measurement of net nutrient flux (blood flow multiplied by venous-arterial concentration difference (VA)) across PDV and liver. Blood flow was measured by downstream dilution of $p$-aminohippurate $(\mathrm{PAH} ; 100 \mathrm{~g} / \mathrm{l})$ infused continuously $(6343 \mathrm{mg} / \mathrm{h})$ into a distal mesenteric vein following a priming dose $(2 \mathrm{~g})$ at 06.00 hours. Immediately following the 4th blood sample set, a $24 \mathrm{~h}$ infusion of $\mathrm{L}$-alanine $(75 \mathrm{mmol} / \mathrm{h})$ was begun into the same mesenteric vein catheter used for $\mathrm{PAH}$ infusion. Infusion of $\mathrm{PAH}$ and blood sampling continued uninterrupted at $30 \mathrm{~min}$ intervals until five additional blood sample sets were obtained. Infusion of PAH was stopped after the 9th blood sample set was drawn. The following day, PAH infusion and blood sampling was repeated beginning at the same times and using similar procedures. After the 4th blood sample set was drawn on day 2, alanine infusion was stopped and $\mathrm{PAH}$ infusion was continued until three more simultaneous 
Table 1. Composition of experimental diets ( $\mathrm{g} / \mathrm{kg}$ dry matter*)

\begin{tabular}{lcc}
\hline $\begin{array}{l}\text { Diet }(\mathrm{g} / \mathrm{kg}) \ldots \\
\text { Ingredient }\end{array}$ & $\begin{array}{c}750 \text { Lucerne } \\
\text { (Medicago sativa })\end{array}$ & 750 Concentrate \\
\hline $\begin{array}{l}\text { Ground lucerne hay } \\
\text { Ground maize }\end{array}$ & $745 \cdot 0$ & $245 \cdot 0$ \\
Soya-bean meal $\dagger$ & $210 \cdot 5$ & $632 \cdot 5$ \\
Dicalcium phosphate & $37 \cdot 0$ & $110 \cdot 0$ \\
Limestone & $2 \cdot 5$ & - \\
Trace-mineralized salt & - & $5 \cdot 5$ \\
\hline
\end{tabular}

* Dry matter content averaged $890 \mathrm{~g} / \mathrm{kg}$.

$\dagger$ Containing $490 \mathrm{~g}$ crude protein (nitrogen $\times 6 \cdot 25$ ) $/ \mathrm{kg}$.

$\ddagger$ Containing $(\mathrm{g} / \mathrm{kg})$ : sodium 370 , chloride 580 , zinc $3 \cdot 5$, iron $3 \cdot 4$, manganese $2 \cdot 0$, copper $0 \cdot 33$, iodide $0 \cdot 07$, cobalt 0.05 , selenium 0.055 .

blood sample sets were obtained at 30 min intervals beginning $1.5 \mathrm{~h}$ after cessation of L-alanine infusion. Thus, blood sample sets were obtained during four periods relative to alanine infusion: immediately before beginning (infusion period 1), immediately after beginning (infusion period 2), immediately before ending (infusion period 3) and starting $1.5 \mathrm{~h}$ after ending (infusion period 4 ) alanine infusion. Within each period, additional blood sample sets were drawn at $15 \mathrm{~min}$ intervals for hormone analysis which will be reported in a subsequent publication.

Alanine solution was infused using $500 \mathrm{ml}$ syringes (Model S-500, Hamilton Co., Reno, NV) and a screw-driven syringe pump (Model 2260, Harvard Apparatus, South Natick, MA) set to deliver $1.2 \mathrm{ml} / \mathrm{min}$. The unbuffered alanine solution infused was prepared the evening before infusions began using commercially prepared L-alanine (Sigma Chemical Co., St Louis, MO) and high-performance liquid chromatography grade water and filtered through a $0.22 \mu \mathrm{m}$ filter (Millipore Corp., Bedford, MA) into sterile containers. Alanine solution was kept refrigerated $\left(2^{\circ}\right)$ until used and, as for PAH solution, was filtered through a $0.45 \mu \mathrm{m}$ filter (Millipore Corp.) when infused into the mesenteric vein catheter.

Blood samples obtained were immediately placed on ice and analysed for blood packed cell volume (PCV), PAH, L-alanine, AAN, ammonia-N, UN and $\beta$-hydroxybutyrate (BOHB), and plasma PAH, glucose and L-lactate as described by Reynolds et al. $(1988 a, b)$. L-alanine was measured in the alkalinized supernatant fraction from perchlorate deproteinization stored at $-20^{\circ}$ using procedures of Williamson (1974). Other analyses were conducted as soon as possible on the day of sampling.

\section{Calculations and statistical analyses}

Calculations of net PDV, liver and total splanchnic nutrient flux, liver extraction ratio and the maximal contributions of precursors removed by liver to liver glucose release were as described by Reynolds et al. (1991). Measurements of visceral nutrient metabolism during infusion periods 2 and 4 were obtained in an effort to describe the relative rate of response to addition or removal of alanine. Because infusion periods 2 and 4 represent acute responses and contain unequal sampling times and because alanine infusion did not follow a priming dose, effects of alanine infusion were tested statistically using infusion periods 1 and 3 only. We feel this represents the best approach for determining effects of additional alanine supply under 'steady-state' conditions. Average rates for each infusionperiod were analysed statistically using general linear models procedures of the Statistical Analysis System (SAS, 1988) and a model testing effects of heifer, diet, infusion-period and diet by infusion-period interaction. Day 2 measurements were not obtained for one heifer 
Table 2. Arterial packed cell volume $(P C V)$ and blood flow $(l / h)$ for the portal vein and liver of beef heifers fed on $750 \mathrm{~g}$ lucerne (Medicago sativa) or $750 \mathrm{~g}$ concentrate $/ \mathrm{kg}$ diets immediately before beginning (Pre) and ending $(+A l a)$ a $24 \mathrm{~h}$ mesenteric vein L-alanine infusion $(75 \mathrm{mmol} / \mathrm{h})^{*}$

\begin{tabular}{|c|c|c|c|c|c|c|c|c|}
\hline \multirow[t]{2}{*}{ Diet $(g / k g) \ldots$} & \multicolumn{2}{|c|}{750 Lucerne } & \multicolumn{2}{|c|}{750 Concentrate } & \multirow[b]{2}{*}{ SE } & \multicolumn{3}{|c|}{$\begin{array}{c}\text { Statistical significance }(P) \\
\text { of effect of: }\end{array}$} \\
\hline & Pre & + Ala & Pre & + Ala & & Diet $†$ & Ala: & Diet $\times$ Ala $\S$ \\
\hline Arterial PCV & $0 \cdot 322$ & 0.313 & $0 \cdot 300$ & $0 \cdot 287$ & 0.013 & 0.074 & $0 \cdot 375$ & $0 \cdot 866$ \\
\hline Portal vein & 714 & 752 & 634 & 632 & 60 & 0.096 & 0.745 & 0.712 \\
\hline Hepatic & 858 & 778 & 777 & 726 & 51 & $0 \cdot 176$ & $0 \cdot 180$ & 0.753 \\
\hline
\end{tabular}

* For details of diets and procedures, see Table I and pp. 438-439.

+ Probability level corresponding to the hypothesis of no diet effect.

+ Probability level corresponding to the hypothesis of no alanine infusion effect.

$\$$ Probability level corresponding to the hypothesis of no diet by alanine infusion interaction.

because of automatic feeder failure; therefore, the experimental data set includes fifteen of sixteen possible observations (four heifers, two diets, two infusion periods).

Average rates of net alanine and AAN metabolism for all four infusion periods are presented graphically to depict the changes induced by abrupt addition and removal of alanine. Values shown are means with their standard errors of average rates for each heifer and diet. Using VA in calculations of net flux, negative values denote net removal (utilization) and positive values denote net absorption or release (production).

\section{RESULTS}

Blood sampling and alanine infusion had no observable adverse effects and heifers always consumed feed offered without delay. Alanine infusion and blood sampling did not affect PCV (Table 2), but heifers had slightly lower $(P<0 \cdot 10)$ PCV when fed on the $750 \mathrm{~g}$ concentrate $/ \mathrm{kg}$ v. $750 \mathrm{~g}$ lucerne $/ \mathrm{kg}$ diet.

\section{Blood flow}

Blood flow for PDV was lower $(P<0 \cdot 10)$ when heifers were fed on the $750 \mathrm{~g}$ concentrate $/ \mathrm{kg}$ v. $750 \mathrm{~g}$ lucerne $/ \mathrm{kg}$ diet (Table 2). There were no effects of alanine infusion on PDV blood flow. Liver blood flow was not affected by diet or alanine infusion $(P>0 \cdot 18)$, but was numerically lower when heifers were fed on the $750 \mathrm{~g}$ concentrate $/ \mathrm{kg} v .750 \mathrm{~g}$ lucerne $/ \mathrm{kg}$ diet (Table 2). Liver blood flow was numerically lower during alanine infusion than during the pre-alanine infusion period, suggesting a decrease in hepatic artery blood flow, but this effect was not significant. Error in measurements of blood flow obtained using PAH dilution can result from inadequate mixing of blood and $\mathrm{PAH}$, or from improper catheter placement within the splanchnic vasculature (Reynolds et al. 1988a). In the present study, diet and alanine infusion comparisons were made within animals. Therefore, any catheterinduced errors in blood flow measurements would be associated with and accounted for by the heifer component of our statistical model.

\section{Alanine metabolism}

Arterial concentration of alanine was increased $(P<0.01)$ by alanine infusion and was greater $(P<0.01)$ when the $750 \mathrm{~g}$ lucerne $/ \mathrm{kg} \mathrm{v.} 750 \mathrm{~g}$ concentrate $/ \mathrm{kg}$ diet was given (Table $3)$. The increase in arterial concentration of alanine resulting from alanine infusion was 
Table 3. Arterial concentrations and venous-arterial concentration differences for nutrients across viscera of beef heifers fed on $750 \mathrm{~g}$ lucerne (Medicago sativa) or $750 \mathrm{~g}$ concentrate/ $\mathrm{kg}$ immediately before beginning (Pre) and ending $(+$ Ala $)$ a $24 \mathrm{~h}$ mesenteric vein infusion of L-alanine $(75 \mathrm{mmol} / \mathrm{h})^{*}$

\begin{tabular}{|c|c|c|c|c|c|c|c|c|}
\hline \multirow[t]{2}{*}{ Diet $(\mathrm{g} / \mathrm{kg}) \ldots$} & \multicolumn{2}{|c|}{750 Lucerne } & \multicolumn{2}{|c|}{750 Concentrate } & \multirow[b]{2}{*}{$\mathrm{SE}$} & \multicolumn{3}{|c|}{$\begin{array}{c}\text { Statistical significance }(P) \\
\text { of effect of: }\end{array}$} \\
\hline & Pre & + Ala & Pre & $+\mathrm{Ala}$ & & Diet & Ala & Diet $\times$ Ala $\S$ \\
\hline \multicolumn{9}{|c|}{ Arterial concentration (mM) } \\
\hline \multicolumn{9}{|c|}{ Blood } \\
\hline L-alanine & $0 \cdot 270$ & 0.608 & $0 \cdot 221$ & 0.478 & $0 \cdot 020$ & $0 \cdot 001$ & 0.001 & $0 \cdot 051$ \\
\hline$x$-Amino-N & $2 \cdot 800$ & $2 \cdot 881$ & $2 \cdot 630$ & $2 \cdot 703$ & $0 \cdot 114$ & $0 \cdot 120$ & U.466 & 0.971 \\
\hline Ammonia- $N$ & $0 \cdot 342$ & 0.408 & $0 \cdot 355$ & 0.448 & $0 \cdot 052$ & 0.577 & $0 \cdot 119$ & 0.785 \\
\hline Urea-N & $12 \cdot 479$ & $15 \cdot 057$ & $10 \cdot 422$ & $11 \cdot 487$ & $0 \cdot 869$ & 0.006 & $0 \cdot 044$ & $0 \cdot 350$ \\
\hline$\beta$-hydroxybutyrate & $0 \cdot 659$ & $0 \cdot 638$ & 0.536 & 0.508 & $0 \cdot 037$ & $0 \cdot 004$ & 0.468 & 0.913 \\
\hline \multicolumn{9}{|l|}{ Plasma } \\
\hline L-iactate & 0.674 & 0.754 & 0.476 & 0.532 & $0 \cdot 044$ & $0 \cdot 001$ & 0.119 & 0.764 \\
\hline Glucose & $4 \cdot 211$ & $4 \cdot 218$ & $4 \cdot 434$ & $4 \cdot 161$ & $0 \cdot 092$ & $0 \cdot 334$ & $0 \cdot 139$ & $0 \cdot 122$ \\
\hline \multicolumn{9}{|l|}{ Portal-arterial (mM) } \\
\hline \multicolumn{9}{|l|}{ Blood } \\
\hline L-alanine & $0 \cdot 040$ & $0 \cdot 153$ & 0.049 & $0 \cdot 176$ & $0 \cdot 014$ & $0 \cdot 220$ & 0.001 & 0.576 \\
\hline$\alpha$-Amino-N & $0 \cdot 168$ & 0.266 & 0.204 & 0.312 & 0.038 & 0.253 & $0 \cdot 014$ & 0.895 \\
\hline Ammonia- $N$ & $0 \cdot 480$ & 0.545 & $0 \cdot 340$ & 0.374 & 0.038 & 0.002 & $0 \cdot 170$ & 0.647 \\
\hline Urea-N & $-0-246$ & $-0 \cdot 344$ & -0.304 & -0.262 & 0.041 & 0.752 & 0.459 & 0.086 \\
\hline$\beta$-hydroxybutyrate & $0 \cdot 198$ & $0 \cdot 211$ & $0 \cdot 176$ & 0.190 & 0.017 & $0 \cdot 194$ & 0.418 & 0.977 \\
\hline \multicolumn{9}{|l|}{ Plasma } \\
\hline L-lactate & $0 \cdot 139$ & $0 \cdot 131$ & 0.125 & 0.113 & 0.013 & 0.223 & $0 \cdot 406$ & 0.880 \\
\hline Glucose & 0.049 & $0 \cdot 020$ & $0 \cdot 182$ & $0 \cdot 153$ & 0.044 & 0.009 & 0.475 & 0.992 \\
\hline \multicolumn{9}{|l|}{ Hepatic-portal (mM) } \\
\hline \multicolumn{9}{|l|}{ Blood } \\
\hline L-alanine & -0.056 & $-0 \cdot 128$ & -0.051 & $-0 \cdot 147$ & $0 \cdot 011$ & $0 \cdot 471$ & $0 \cdot 001$ & $0 \cdot 260$ \\
\hline$\alpha$-Amino- $N$ & -0.139 & -0.230 & $-0 \cdot 164$ & -0.245 & 0.027 & 0.429 & 0.007 & 0.840 \\
\hline Ammonia-N & -0.487 & -0.554 & -0.344 & $-0 \cdot 374$ & $0 \cdot 042$ & 0.002 & $0 \cdot 218$ & 0.628 \\
\hline Urea-N & 0.693 & 0.962 & $0-566$ & $0 \cdot 700$ & 0.035 & 0.001 & $0 \cdot 001$ & 0.058 \\
\hline$\beta$-hydroxybutyrate & 0.097 & 0.072 & $0 \cdot 110$ & $0 \cdot 084$ & 0.015 & $0 \cdot 359$ & 0.088 & 0.982 \\
\hline \multicolumn{9}{|l|}{ Plasma } \\
\hline L-lactate & -0.079 & 0.009 & -0.077 & 0.000 & 0.030 & $0 \cdot 898$ & $0 \cdot 014$ & 0.853 \\
\hline Glucose & $0 \cdot 360$ & $0 \cdot 380$ & $0 \cdot 366$ & 0.399 & 0.021 & 0.522 & $0 \cdot 195$ & 0.744 \\
\hline \multicolumn{9}{|l|}{ Hepatic-arterial (mм) } \\
\hline \multicolumn{9}{|l|}{ Blood } \\
\hline L-alanine & -0.016 & 0.025 & -0.022 & $0-029$ & $0 \cdot 010$ & $0 \cdot 330$ & 0.002 & $0 \cdot 573$ \\
\hline$\alpha$-Amino-N & 0.028 & 0.036 & 0.040 & 0.067 & 0.020 & $0 \cdot 252$ & 0.339 & 0.577 \\
\hline Ammonia- $\mathrm{N}$ & -0.007 & -0.009 & -0.004 & 0.001 & 0.007 & 0.348 & 0.863 & 0.609 \\
\hline Urea-N & $0 \cdot 448$ & 0.618 & $0 \cdot 262$ & 0.438 & $0 \cdot 050$ & $0 \cdot 003$ & 0.004 & $0 \cdot 950$ \\
\hline$\beta$-hydroxybutyrate & 0.295 & $0 \cdot 283$ & 0.286 & $0 \cdot 274$ & $0 \cdot 014$ & $0 \cdot 477$ & 0.353 & 0.990 \\
\hline \multicolumn{9}{|l|}{ Plasma } \\
\hline L-lactate & $0 \cdot 060$ & $0 \cdot 139$ & 0.048 & $0 \cdot 113$ & $0 \cdot 027$ & 0.449 & $0 \cdot 017$ & 0.781 \\
\hline Glucose & 0.409 & 0.400 & 0.549 & 0.552 & 0.049 & 0.010 & 0.955 & 0.893 \\
\hline
\end{tabular}

* For details of diets and procedures, see Table 1 and pp. 438-439.

$\uparrow$ Probability level corresponding to the hypothesis of no diet effect.

* Probability level corresponding to the hypothesis of no alanine infusion effect.

$\$$ Probability level corresponding to the hypothesis of no diet by alanine infusion interaction.

greater when heifers were fed on the $750 \mathrm{~g}$ lucerne $/ \mathrm{kg} v .750 \mathrm{~g}$ concentrate $/ \mathrm{kg}$ diet $(P<$ 0.05). Portal-arterial and hepatic-arterial VA for alanine were increased $(P<0.01$; became more positive) and hepatic-portal VA for alanine were decreased $(P<0.01$; became more negative) by alanine infusion. Net PDV release and liver removal of alanine were increased 
$(P<0.01)$ by alanine infusion (Table 4$)$. On average, net PDV release of alanine increased $83 \mathrm{mmol} / \mathrm{h}$, which is reassuringly close to the amount infused $(75 \mathrm{mmol} / \mathrm{h})$.

The amount of alanine infused was based on previous data from these heifers (Reynolds et al. 1991) fed on low and high intakes of the diets given in the present study. We gave relatively low intakes and infused $75 \mathrm{mmol} / \mathrm{h}$ in attempting to achieve a net PDV release of $100 \mathrm{mmol} / \mathrm{h}$, which is twice the net PDV release measured for high intake in the previous study. We exceeded this rate by $12 \mathrm{mmol} / \mathrm{h}$ (Table 4 ). The increase in liver removal of alanine during alanine infusion averaged $54 \mathrm{mmol} / \mathrm{h}$; therefore, the infusion rate chosen plus basal net PDV release exceeded the maximal capacity of the liver to remove alanine under these experimental conditions $(92 \mathrm{mmol} / \mathrm{h})$.

The percentage of net PDV release of alanine removed by liver decreased $(P<0.05)$ from $132 \%$ before alanine infusion began to $80 \%$ during alanine infusion (Table 5 ). It is possible that liver alanine removal could have increased with longer alanine infusion; however, increases in liver removal of alanine appeared due to increases in portal supply and not to direct effects on liver transport systems, because liver alanine extraction ratio (the percentage of total liver supply removed) was not altered by alanine infusion (Table 5). Negative rates of net total splanchnic alanine flux reflect net utilization of alanine from peripheral tissues by the liver. Because the increase in liver alanine removal was less than the increase in net PDV alanine release resulting from alanine infusion, net total splanchnic flux of alanine switched $(P<0.01)$ from net removal to net release (Table 4$)$ and arterial concentration of alanine increased (Table 3). Diet had no effects on VA (Table 3) or net flux of alanine across PDV and liver (Table 4).

\section{AAN metabolism}

Arterial AAN concentration was not affected by alanine infusion, in spite of the increase in arterial alanine concentration (Table 3). Portal-arterial VA for AAN were increased $(P<0.05)$ and hepatic-portal VA for AAN were decreased $(P<0.01)$ by alanine infusion (Table 3). On average, net PDV release of AAN was increased $(P<0.01) 74 \mathrm{mmol} / \mathrm{h}$ by alanine infusion (Table 4).

Net liver removal of AAN was increased $(P<0.01) 62 \mathrm{mmol} / \mathrm{h}$ by alanine infusion (Table 4). Net total splanchnic release of AAN was not significantly affected by alanine infusion, but was numerically greater when alanine was infused. Liver extraction ratio for AAN was increased $(P<0.01)$ by alanine infusion, which is in contrast to the lack of response of liver extraction ratio for alanine (Table 5). Liver removal of AAN as a percentage of net PDV release was not affected by alanine infusion (Table 5), but was numerically lower when heifers were fed on the $750 \mathrm{~g}$ concentrate $/ \mathrm{kg} v .750 \mathrm{~g}$ lucerne $/ \mathrm{kg}$ diet. This difference was more evident $(P<0.05)$ when data from all four periods were included in the statistical analysis. In our previous study (Reynolds et al. 1991), liver removal of AAN was greater in heifers fed on the $750 \mathrm{~g}$ lucerne $/ \mathrm{kg} v .750 \mathrm{~g}$ concentrate $/ \mathrm{kg}$ diet and this difference was associated with greater liver removal of $\mathrm{NH}_{3}-\mathrm{N}$.

\section{$\mathrm{NH}_{3}-\mathrm{N}$ metabolism}

Arterial concentration of $\mathrm{NH}_{3}-\mathrm{N}$ was not affected by diet, but tended $(P<0 \cdot 12)$ to be greater when alanine was infused (Table 3). Portal-arterial VA for $\mathrm{NH}_{3}-\mathrm{N}$ were greater $(P<0.01)$ and hepatic-portal VA for $\mathrm{NH}_{3}-\mathrm{N}$ were lower $(P<0.01)$ when heifers were fed on the $750 \mathrm{~g}$ lucerne $/ \mathrm{kg}$ v. $750 \mathrm{~g}$ concentrate $/ \mathrm{kg}$ diet (Table 3). Net $\mathrm{NH}_{3}-\mathrm{N}$ absorption by PDV and removal by the liver was greater $(P<0.01)$ in heifers fed on the $750 \mathrm{~g}$ lucerne $/ \mathrm{kg} v .750 \mathrm{~g}$ concentrate $/ \mathrm{kg}$ diet (Table 4 ).

Increases in net PDV absorption $(P<0 \cdot 12)$ and liver removal $(P<0 \cdot 16)$ of $\mathrm{NH}_{3}-\mathrm{N}$ during alanine infusion were not significant, but were numerically greater when heifers were 
Table 4. Net flux of nutrients (mmol/h) across visceral tissues of beef heifers fed on $750 \mathrm{~g}$ lucerne (Medicago sativa) or $750 \mathrm{~g}$ concentrate/ $\mathrm{kg}$ immediately before beginning (Pre) and ending $(+$ Ala $)$ a $24 \mathrm{~h}$ mesenteric vein infusion of L-alanine $(75 \mathrm{mmol} / \mathrm{h})^{*}$

\begin{tabular}{|c|c|c|c|c|c|c|c|c|}
\hline \multirow{2}{*}{ Diet $(\mathrm{g} / \mathrm{kg}) \ldots$} & \multicolumn{2}{|c|}{750 Lucerne } & \multicolumn{2}{|c|}{750 Concentrate } & \multirow[b]{2}{*}{$\mathrm{SE}$} & \multicolumn{3}{|c|}{$\begin{array}{c}\text { Statistical significance }(P) \\
\text { of effect of: }\end{array}$} \\
\hline & Pre & $+\mathrm{Ala}$ & Pre & + Ala & & Diet $\uparrow$ & Ala $\ddagger$ & Diet $\times$ Ala $\S$ \\
\hline \multicolumn{9}{|l|}{ Portal-drained viscera } \\
\hline \multicolumn{9}{|l|}{ Blood } \\
\hline L-alanine & 28 & 116 & 30 & 109 & 5 & 0.542 & $0-001$ & $0 \cdot 286$ \\
\hline$\alpha$-Amino-N & 118 & 200 & 127 & 192 & 17 & 0.945 & $0 \cdot 00 \mathrm{I}$ & 0.582 \\
\hline Urea-N & -177 & -264 & -194 & -166 & 44 & $0 \cdot 320$ & 0.463 & 0.171 \\
\hline Ammonia-N & 344 & 408 & 214 & 233 & 26 & 0.001 & $0 \cdot 113$ & $0 \cdot 362$ \\
\hline$\beta$-hydroxybutyrate & 141 & 161 & 112 & 118 & 13 & 0.015 & $0 \cdot 230$ & 0.565 \\
\hline \multicolumn{9}{|l|}{ Plasma } \\
\hline L-lactate & 62 & 62 & 52 & 47 & 5 & 0.027 & 0.576 & 0.557 \\
\hline Glucose & 21 & 10 & 71 & 62 & 14 & 0.003 & 0.430 & 0.938 \\
\hline \multicolumn{9}{|l|}{ Liver } \\
\hline \multicolumn{9}{|l|}{ Blood } \\
\hline L-alanine & -43 & -95 & -32 & -88 & 9 & $0 \cdot 273$ & 0.001 & 0.849 \\
\hline$\alpha$-Amino- $N$ & -95 & -171 & -98 & -145 & 13 & 0.332 & 0.001 & $0 \cdot 248$ \\
\hline Urea-N & 563 & 743 & 388 & 480 & 46 & 0.001 & $0 \cdot 010$ & $0 \cdot 304$ \\
\hline Ammonia-N & -350 & -414 & -217 & -232 & 28 & 0.001 & 0.151 & $0 \cdot 366$ \\
\hline$\beta$-hydroxybutyrate & 112 & 59 & 111 & 81 & 20 & 0.562 & 0.042 & 0.516 \\
\hline \multicolumn{9}{|l|}{ Plasma } \\
\hline L-lactate & -29 & 10 & -29 & 7 & 15 & 0.932 & 0.020 & $0 \cdot 880$ \\
\hline Glucose & 192 & 195 & 214 & 203 & 14 & $0 \cdot 260$ & 0.727 & 0.572 \\
\hline \multicolumn{9}{|l|}{ Total splanchnic } \\
\hline \multicolumn{9}{|l|}{ Blood } \\
\hline L-alanine & -15 & 20 & -2 & 21 & 8 & $0 \cdot 365$ & $0 \cdot 003$ & $0 \cdot 391$ \\
\hline$\alpha$-Amino-N & 22 & 28 & 30 & 47 & 13 & $0 \cdot 301$ & $0 \cdot 347$ & 0.634 \\
\hline Urea-N & 386 & 479 & 194 & 314 & 45 & 0.002 & 0.028 & $0 \cdot 744$ \\
\hline Ammonia- $N$ & -6 & -7 & -3 & 1 & 6 & $0 \cdot 329$ & 0.781 & $0 \cdot 722$ \\
\hline$\beta$-hydroxybutyrate & 253 & 220 & 223 & 199 & 19 & 0.160 & 0.125 & 0.787 \\
\hline \multicolumn{9}{|l|}{ Plasma } \\
\hline L-lactate & 32 & 72 & 24 & 54 & 14 & $0 \cdot 300$ & 0.019 & $0 \cdot 700$ \\
\hline Glucose & 214 & 205 & 286 & 265 & 14 & 0.001 & $0 \cdot 272$ & 0.632 \\
\hline
\end{tabular}

* For details of diets and procedures, see Table $\mathrm{I}$ and pp. 438439 .

$\dagger$ Probability level corresponding to the hypothesis of no diet effect.

* Probability level corresponding to the hypothesis of no alanine infusion effect.

$\S$ Probability level corresponding to the hypothesis of no diet by alanine infusion interaction.

fed on the $750 \mathrm{~g}$ lucerne $/ \mathrm{kg} v .750 \mathrm{~g}$ concentrate $/ \mathrm{kg}$ diet (Table 4). This trend towards increased net PDV release of $\mathrm{NH}_{3}-\mathrm{N}$ could be due to increased transfer of $\mathrm{UN}$ into the gut. Liver extraction ratio for $\mathrm{NH}_{3}-\mathrm{N}$ (Table 5) was greater $(P<0.01)$ when heifers were fed on the $750 \mathrm{~g}$ lucerne $/ \mathrm{kg} v$. the $750 \mathrm{~g}$ concentrate $/ \mathrm{kg}$ diet, which agrees with previous observations for these diets (Reynolds et al. 1991).

\section{UN metabolism}

Arterial UN concentration (Table 3) was greater when heifers were fed on the $750 \mathrm{~g}$ lucerne $/ \mathrm{kg} v .750 \mathrm{~g}$ concentrate $/ \mathrm{kg}$ diet $(P<0.01)$ and greater when alanine was infused $(P<0.05)$. Portal-arterial VA for UN (Table 3$)$ were decreased by alanine infusion when heifers were fed on the $750 \mathrm{~g}$ lucerne $/ \mathrm{kg}$ diet and increased by alanine infusion when heifers were fed on the $750 \mathrm{~g}$ concentrate $/ \mathrm{kg}$ diet $(P<0 \cdot 10)$. Hepatic - portal and hepatic-arterial VA for UN were greater $(P<0.01)$ when heifers were fed on the $750 \mathrm{~g}$ lucerne $/ \mathrm{kg} v .750 \mathrm{~g}$ 
Table 5. Proportion of nutrients extracted by the liver in beef heifers fed on $750 \mathrm{~g}$ lucerne (Medicago sativa) or $750 \mathrm{~g}$ concentrate immediately before beginning (Pre) and ending $(+$ Ala $)$ a 24 mesenteric vein infusion of $L$-alanine $(75 \mathrm{mmol} / \mathrm{h})^{*}$

\begin{tabular}{|c|c|c|c|c|c|c|c|c|}
\hline \multirow{2}{*}{$\begin{array}{l}\operatorname{Diet}(\mathrm{g} / \mathrm{kg}) \ldots \\
\text { item }\end{array}$} & \multicolumn{2}{|c|}{750 Lucerne } & \multicolumn{2}{|c|}{750 Concentrate } & \multirow[b]{2}{*}{ SE } & \multicolumn{3}{|c|}{$\begin{array}{c}\text { Statistical significance }(P) \\
\text { of effect of: }\end{array}$} \\
\hline & Pre & + Ala & Pre & + Ala & & Diet & Ala $\ddagger$ & Diet $\times$ Ala \\
\hline \multicolumn{9}{|l|}{ Liver extraction } \\
\hline l-alanine & $0 \cdot 164$ & $0 \cdot 161$ & $0 \cdot 161$ & $0 \cdot 193$ & 0.023 & $0-296$ & 0.719 & $0 \cdot 386$ \\
\hline$\alpha$-Amino nitrogen & 0.038 & $0 \cdot 070$ & $0 \cdot 046$ & $0 \cdot 068$ & $0 \cdot 004$ & $0 \cdot 153$ & 0.001 & $0 \cdot 022$ \\
\hline Ammonia nitrogen & 0.548 & 0.570 & $0 \cdot 452$ & 0.426 & 0.051 & 0.003 & 0.963 & 0.739 \\
\hline L-lactate & 0.069 & $(+0 \cdot 29)$ & $0 \cdot 100$ & $(+0 \cdot 11)$ & $0 \cdot 048$ & $0 \cdot 334$ & $0 \cdot 052$ & 0.986 \\
\hline \multicolumn{9}{|c|}{ Ratio nutrients removed by liver: released by portal-drained viscera } \\
\hline L-alanine & 1.569 & 0.806 & $1 \cdot 069$ & 0.808 & $0 \cdot 272$ & $0 \cdot 040$ & $0 \cdot 002$ & $0 \cdot 124$ \\
\hline$\alpha$-Amino nitrogen & 0.829 & $0 \cdot 855$ & 0.780 & 0.763 & 0.073 & $0 \cdot 019$ & 0.045 & $0 \cdot 482$ \\
\hline Ammonia nitrogen & 1.016 & $1 \cdot 021$ & $0 \cdot 014$ & 0.998 & $0 \cdot 017$ & $0 \cdot 613$ & 0.479 & $0 \cdot 600$ \\
\hline L-lactate & 0.456 & $(+0.215)$ & $0.54 I$ & $(+0 \cdot 260)$ & 0.296 & 0.770 & 0.024 & 0.970 \\
\hline
\end{tabular}

\footnotetext{
* For details of diets and procedures, see Table 1 and pp. 438-439.

$\dagger$ Probability level corresponding to the hypothesis of no diet effect.

* Probability level corresponding to the hypothesis of no alanine infusion effect.

$\S$ Probability level corresponding to the hypothesis of no diet by alanine infusion interaction.
}

Table 6. Maximum possible contribution of precurvors removed to liver glucose carbon released in beef heifers fed on $750 \mathrm{~g}$ lucerne (Medicago sativa) or $750 \mathrm{~g}$ concentrate $/ \mathrm{kg}$ immediately before beginning (Pre) and ending $(+A l a)$ a 24 h mesenteric vein infusion of $L^{-}$ alanine $(75 \mathrm{mmol} / \mathrm{h})^{*}$

\begin{tabular}{|c|c|c|c|c|c|c|c|c|}
\hline \multirow{2}{*}{$\begin{array}{l}\operatorname{Diet}(\mathrm{g} / \mathrm{kg}) \ldots \\
\text { Item }\end{array}$} & \multicolumn{2}{|c|}{750 Lucerne } & \multicolumn{2}{|c|}{750 Concentrate } & \multirow[b]{2}{*}{$\mathrm{SE}$} & \multicolumn{3}{|c|}{$\begin{array}{c}\text { Statistical significance }(P) \\
\text { of effect of: }\end{array}$} \\
\hline & Pre & + Ala & Pre & + Ala & & Diet $t$ & Alaf & Diet $\times$ Alas \\
\hline L-alanine & $0 \cdot 108$ & $0 \cdot 239$ & $0 \cdot 078$ & $0 \cdot 223$ & $0 \cdot 021$ & $0 \cdot 251$ & 0.001 & $0 \cdot 731$ \\
\hline$\alpha$-Amino nitrogen & $0 \cdot 260$ & $0 \cdot 430$ & $0 \cdot 235$ & $0 \cdot 365$ & 0.031 & $0-139$ & 0.001 & 0.476 \\
\hline L-Lactate & $0 \cdot 082$ & $(-0.019)$ & $0 \cdot 066$ & $(-0.026)$ & 0.039 & 0.753 & $0-022$ & 0.898 \\
\hline$\alpha$-Amino nitrogen + L-lactate & $0 \cdot 342$ & 0.411 & $0 \cdot 302$ & 0.339 & $0 \cdot 041$ & $0 \cdot 156$ & $0 \cdot 172$ & 0.592 \\
\hline
\end{tabular}

* For details of diet and procedures, see Table 1 and pp. 438.439.

$\uparrow$ Probability level corresponding to the hypothesis of no diet effect.

¥ Probability level corresponding to the hypothesis of no alanine infusion effect.

$\S$ Probability level corresponding to the hypothesis of no diet by alanine infusion interaction.

concentrate $/ \mathrm{kg}$ diet and were increased $(P<0 \cdot 01)$ by alanine infusion, but the increase in hepatic-portal VA for UN resulting from alanine infusion was greater $(P<0.05)$ when heifers were fed on the $750 \mathrm{~g}$ lucerne $/ \mathrm{kg} v .750 \mathrm{~g}$ concentrate $/ \mathrm{kg}$ diet (Table 3). Net PDV removal of UN from arterial blood was not affected $(P>0 \cdot 10)$ by diet or alanine infusion. This measurement of UN transfer into PDV does not account for transfer via saliva. Net liver and total splanchnic release of UN were greater $(P<0.01)$ when heifers were fed on the $750 \mathrm{~g}$ lucerne $/ \mathrm{kg} v .750 \mathrm{~g}$ concentrate $/ \mathrm{kg}$ diet (Table 4). Both net liver $(P<0.01)$ and total splanchnic $(P<0.05)$ release of UN were increased by alanine infusion, which explains the observed increases in arterial UN concentration during alanine infusion (Table 3). 
Increases in liver UN release resulting from alanine infusion (180 and $92 \mathrm{mmol} / \mathrm{h}$ for the $750 \mathrm{~g}$ lucerne $/ \mathrm{kg}$ and $750 \mathrm{~g}$ concentrate $/ \mathrm{kg}$ diets respectively) are greater than the measured increases in net liver removal of alanine or AAN. This was especially true when heifers were fed on the lucerne diet (Table 4). Increases in liver removal of $\mathrm{NH}_{3}-\mathrm{N}$ also contributed to the increase in liver UN release during alanine infusion.

\section{BOHB metabolism}

Arterial concentration of $\mathrm{BOHB}$ was greater $(P<0.01)$ when heifers were fed on the $750 \mathrm{~g}$ lucerne $/ \mathrm{kg}$ v. $750 \mathrm{~g}$ concentrate $/ \mathrm{kg}$ diet (Table 3 ). Portal-arterial and hepatic-arterial VA for BOHB were not affected by diet or alanine infusion, but hepatic-portal VA for BOHB was decreased $(P<0.10)$ by alanine infusion (Table 3). Net PDV release of BOHB was greater $(P<0.05)$ when heifers were fed on the $750 \mathrm{~g}$ lucerne $/ \mathrm{kg} v .750 \mathrm{~g}$ concentrate $/ \mathrm{kg}$ diet (Table 4), which was observed previously (Reynolds et al. 1991). Net liver release of BOHB was decreased $(P<0 \cdot 05)$ by alanine infusion (Table 4$)$, but the decrease in net total splanchnic release of BOHB during alanine infusion was not significant $(P>0 \cdot 13)$.

\section{Lactate metabolism}

Lactate concentration in arterial plasma was greater $(P<0.01)$ when heifers were fed on the $750 \mathrm{~g}$ lucerne $/ \mathrm{kg} \mathrm{v} .750 \mathrm{~g}$ concentrate $/ \mathrm{kg}$ diet (Table 3 ). In the previous study (Reynolds et al. 1991), arterial lactate concentration was greater in heifers fed on the $750 \mathrm{~g}$ lucerne $/ \mathrm{kg}$ v. $750 \mathrm{~g}$ concentrate $/ \mathrm{kg}$ diet at low, but not high intake. Lactate VA were not affected by diet, but hepatic portal $(P<0.05)$ and hepatic-arterial $(P<0.01)$ VA for lactate were increased by alanine infusion (Table 3). Net PDV release of lactate was greater $(P<0.05)$ when heifers were fed on the $750 \mathrm{~g}$ lucerne $/ \mathrm{kg} \mathrm{v.} 750 \mathrm{~g}$ concentrate $/ \mathrm{kg}$ diet. Net flux of lactate across liver switched $(P<0.05)$ from net removal during the pre-infusion period to net release during alanine infusion (Table 4$)$, which resulted in an increase $(P<$ 0.05 ) in net total splanchnic release of lactate. Assuming all the increase in net liver flux of lactate during alanine infusion represents a conversion of alanine to lactate via pyruvate (Hetenyi et al. 1980), the change in net liver flux of lactate $(37 \mathrm{mmol} / \mathrm{h}$ ) accounts for $69 \%$ of the increase in net liver removal of alanine (Table 4). It is also possible that the decrease in liver lactate removal during alanine infusion resulted from a decrease in lactate uptake or its use in metabolic processes, or both, rather than an increase in lactate synthesis from pyruvate.

\section{Glucose metabolism}

Portal-arterial and hepatic-arterial VA for glucose (Table 3) and net PDV and total splanchnic release of glucose (Table 4$)$ were greater $(P<0.01)$ when heifers were fed on the $750 \mathrm{~g}$ concentrate $/ \mathrm{kg} v .750 \mathrm{~g}$ lucerne $/ \mathrm{kg}$ diet (Table 3 ). Alanine infusion had no effect on net glucose release by PDV or liver (Table 4). Net PDV release of glucose was substantial ( $32 \%$ of net liver glucose release) when heifers were fed on the $750 \mathrm{~g}$ concentrate $/ \mathrm{kg}$ diet and must represent glucose absorption from the diet, which was primarily maize starch. Net PDV glucose absorption was also evident when heifers were fed on the $750 \mathrm{~g}$ lucerne $/ \mathrm{kg}$ diet, which also contained ground maize. In the previous study conducted when these heifers were less mature (Reynolds et al. 1991), net PDV glucose absorption was positive when heifers were fed on concentrate at low, but not high intake.

While net liver release of glucose was not affected by alanine infusion, the maximum theoretical contribution to liver glucose release of alanine and AAN was increased $(P<$ $0.01)$ and of lactate was decreased $(P<0.05)$ by alanine infusion (Table 6). These rates represent maximal estimates of $C$ availability for glucose synthesis, and true rates of glucose synthesis from these precursors will be lower. In sheep, Wolff \& Bergman (1972) found that 

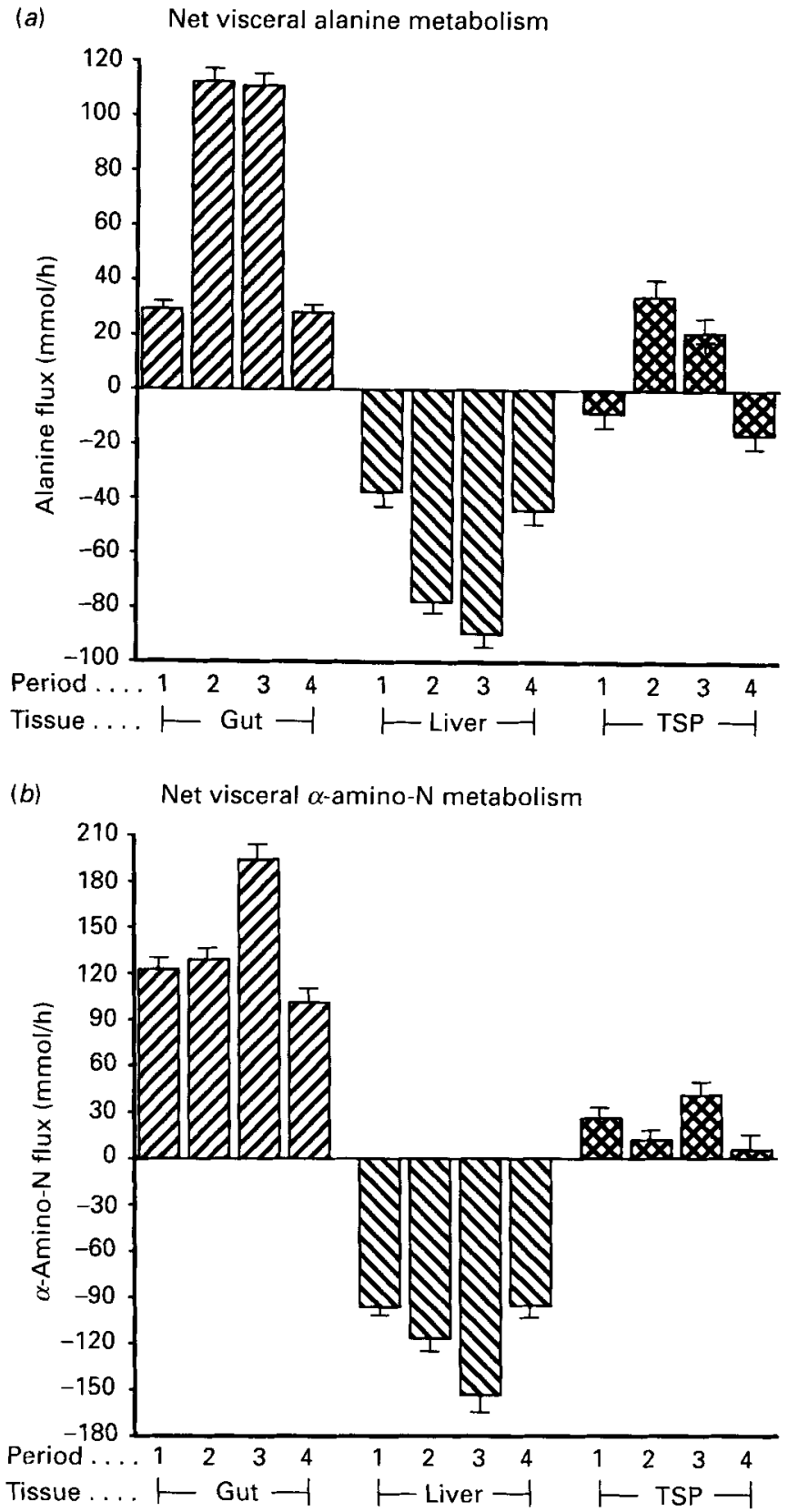

Fig. 1. Net flux of $(a)$ L-alanine and $(b) \alpha$-amino-nitrogen across portal-drained visceral (Gut), liver and total splanchnic (TSP) tissues in beef heifers fed on $750 \mathrm{~g}$ concentrate $/ \mathrm{kg}$ and $750 \mathrm{~g}$ lucerne (Medicago sativa) $/ \mathrm{kg}$ diets immediately before (period 1) and after (period 2) beginning and immediately before (period 3) and $1.5 \mathrm{~h} \mathrm{after}$ (period 4) ending a $24 \mathrm{~h}$ mesenteric vein infusion of L-alanine. Values are least squares means with their standard errors represented by vertical bars for both diets. For details of diets and procedures, see Table 1 and pp. $438-439$. 
the use of $\mathrm{C}$ from ${ }^{14} \mathrm{C}$-labelled alanine for liver glucose synthesis represented $76 \%$ of maximal rates estimated using net flux measurements. In the same study, isotopic measurements of liver glucose synthesis from the seven principal gluconeogenic amino acids represented $38 \%$ of maximal rates estimated using net flux measurements.

\section{Acute v. steady-state responses to alanine infusion}

Arterial concentration of alanine was immediately elevated by alanine infusion from $0.245 \mathrm{~mm}$ (infusion period 1) to $0.446 \mathrm{~mm}$ (infusion period 2) and was further elevated to $0.543 \mathrm{~mm}$ during the last $1.5 \mathrm{~h}$ of alanine infusion (infusion period 3). Arterial concentration of alanine had returned to $0.274 \mathrm{~mm}$ within $1.5 \mathrm{~h}$ after ending alanine infusion (infusion period 4). Increased arterial concentration of alanine during alanine infusion resulted from an immediate increase in net total splanchnic release of alanine (Fig. 1). Net total splanchnic release of alanine was numerically lower during infusion period 3 than infusion period 2 because liver alanine removal increased slightly.

It is surprising that net PDV release of AAN increased during infusion period 3, but not during infusion period 2 (Fig. 1). This implies that, in the short term, net PDV release of AAN other than alanine decreased in response to alanine infusion. Whether this resulted from a decrease in absorption or an increase in utilization of amino acids by PDV is uncertain. This result does suggest an adaptation to alanine infusion occurred after $22 \mathrm{~h}$. In contrast to net PDV release, net liver removal of AAN was numerically increased by alanine infusion during infusion period 2 (Fig. 1).

Effects of alanine infusion on liver release of UN and net PDV absorption and liver removal of $\mathrm{NH}_{3}-\mathrm{N}$ were more evident during infusion period 3 than infusion period 2 (values not shown); however, the increase in liver UN release between infusion periods 1 and 2 was nearly equal to the increase in liver alanine removal. This implies that after $22 \mathrm{~h}$ urea cycle activity increased in response to continued catabolism of alanine and the increase in arterial concentration, net PDV absorption and net liver removal of $\mathrm{NH}_{3}-\mathrm{N}$. For both lactate and $\mathrm{BOHB}$, effects of alanine infusion on their net metabolism by liver were evident during infusion period 2, but the greatest effect was measured during infusion period 3 (values not shown). Liver lactate removal returned to the basal rate during period 4 , but liver BOHB release was still lower during infusion period 4 than during infusion period 1.

\section{DISCUSSION}

The close agreement between the amount of alanine infused and measured increases in net PDV absorption of alanine and AAN lends credence to the measurements obtained. This is especially true when errors associated with measurement of blood flow and VA for these nutrients and inherent day-to-day variation in PDV metabolism are considered. This finding suggests that in these heifers blood from the mesenteric veins was adequately mixed with blood from other portal vein branches before reaching the portal vein sampling site and that streaming of portal vein blood was not a problem.

In general, effects of diet composition on net visceral nutrient metabolism in the present study agree with other measurements obtained from beef cattle fed on these diets at low intakes (Reynolds et al. 1990, 1991). Differences in net PDV glucose absorption between diets can be attributed to differences in maize starch intake, while differences in visceral non-protein- $\mathrm{N}$ metabolism between diets can be attributed to differences in $\mathrm{N}$ intake and digestion (Reynolds \& Tyrrell, 1989). Differences in net PDV glucose flux between the $750 \mathrm{~g}$ lucerne $/ \mathrm{kg}$ and the $750 \mathrm{~g}$ concentrate $/ \mathrm{kg}$ diets in the present study account for $94 \%$ of the calculated difference in small intestinal starch digestion (Owens et al. 1986). 
Greater PDV blood flow when heifers were fed on the $750 \mathrm{~g}$ lucerne $/ \mathrm{kg} v .750 \mathrm{~g}$ concentrate $/ \mathrm{kg}$ diet was observed previously when these diets were given at equal $\mathrm{ME}$ (Reynolds et al. 1991) and was evident in the present study in spite of slightly greater calculated ME intake when heifers were fed on the $750 \mathrm{~g}$ concentrate $/ \mathrm{kg}$ diet. Effects of ME intake on PDV blood flow are well documented in cattle (Lomax \& Baird, 1983). In the present study, alanine infusion supplied an additional $2.93 \mathrm{MJ}$ energy over $24 \mathrm{~h}$, which had no effect on PDV blood flow and tended to decrease liver blood flow. Metabolic activity of PDV is likely to be the primary determinant of PDV blood flow (Shepherd, 1982) and it does not appear that long-term infusion of alanine altered PDV metabolic activity in the present study (Table 4). Together, increased liver removal of AAN and $\mathrm{NH}_{3}-\mathrm{N}$ account for $74 \%$ of the increase in liver UN release during alanine infusion. In the present and in previous studies (Reynolds et al. 1990, 1991), liver $\mathrm{AAN}$ and $\mathrm{NH}_{3}-\mathrm{N}$ removal account for $70-90 \%$ of liver UN release in beef cattle fed on these diets. Other sources of UN such as amino acid $\mathrm{R}$ groups (side chains), nucleic acids and peptides could also contribute to measured increases in liver UN release induced by alanine infusion. In sheep, spatial separation of the urea cycle is evident when net fluxes of urea cycle intermediates are considered (Bergman \& Heitmann, 1978). While all the urea cycle reactions occur in the liver, some of the individual reactions also occur in other tissues. Citrulline and urea are released by liver, ornithine is removed by kidneys and arginine is released by kidneys and removed by liver. This movement of $\mathrm{N}$ via urea cycle intermediates contributes to total urea production by the liver. Assuming this inter-organ transfer of urea cycle intermediates is inherent in cattle, measurements of AAN flux do not account for all the $\mathrm{N}$ flowing to the liver urea cycle as arginine.

The continued elevation of liver UN release and PDV absorption of $\mathrm{NH}_{3}-\mathrm{N}$ during period 4, when liver removal of alanine and AAN had returned to basal rates, is further evidence that the increase in liver UN release is due in part to induction of the urea cycle and an increase in recycling of non-protein- $\mathrm{N}$ through the gut. Inter-organ transfer of urea cycle intermediates could also contribute to a lag in the response of liver UN release to addition and removal of infused alanine.

In vitro, lactate, alanine and pyruvate stimulate ureagenesis in the presence of ornithine, oleate and ammonium chloride to a similar extent (Krebs et al. 1979). This has been attributed to the entry of pyruvate into the tricarboxylic acid cycle and the subsequent formation of ATP, $\alpha$-ketoglutarate, glutamate and aspartate (Krebs et al. 1979; Briggs \& Freedland, 1982). However, in the present study much of the increase in liver UN release induced by alanine infusion may also be due to increased transfer of alanine- $\mathrm{N}$ to aspartate or to carbamoyl phosphate via glutamate, or both (Newsholme \& Leach, 1983), rather than effects of additional pyruvate formation per se.

Depression of ketogenesis by alanine has been observed in rats (Ozand et al. 1977) and humans (Genuth, 1973). The effect of alanine on liver BOHB release has been attributed to alterations of liver NADH:NAD ratio (Ozand et al. 1977), a decrease in fatty acid oxidation (Ozand et al. 1978) and an increase in oxaloacetate availability and citrate formation which reduces acetyl-CoA availability for BOHB formation (Nosandi et al. 1980). These effects appear to follow an increase in pyruvate formation and pyruvate is also antiketogenic in vitro (Demaugre et al. 1982).

In these heifers it is unlikely that glucose precursor availability was limiting, therefore it is not surprising that alanine infusion did not affect net glucose release by liver. The decrease in net liver lactate removal observed during alanine infusion suggests that increased alanine supply to the liver shuffled the availability of glucose precursors. Similar effects on liver lactate removal were reported in dairy cows during mesenteric vein infusion of propionate (Baird et al. 1980). In dogs, alanine infusion into a peripheral vein doubled 
liver alanine removal and the conversion of alanine to glucose without affecting net liver glucose release (Diamond et al. 1988). Measurements of net liver glucose release may not account for changes in liver glycogen metabolism that could have occurred during alanine infusion.

In summary, mesenteric vein infusion of alanine increased the flow of non-protein- $\mathrm{N}$ through liver, decreased liver removal of lactate and release of BOHB, but had no effect on net liver release of glucose in beef heifers. Effects of diet forage:concentrate ratio on net nutrient metabolism by PDV and liver were similar to those observed in a previous study using these heifers (Reynolds et al. 1991). In the present study, net PDV glucose absorption was substantial when heifers were fed on a concentrate diet containing $750 \mathrm{~g}$ maize and soya-bean meal $/ \mathrm{kg}$ at relatively low intake.

The authors express sincere appreciation to the following for contributions to this study: Ben Stroud, Katie McLaughlin Phalen and others for veterinary care of the animals; Animal Operations personnel for daily care of the animals, with special thanks to Kevin Reed, Bob Kreft, Tom Estes, Tom Bunting and Al Thomas; Dennis Hucht, Bob Ransom, Helene Lapierre, Ben Gadsden and others for able help in obtaining and analysing samples; Larry Douglass for statistical advice; and Cindy Hanna, Jean Wilcox, Vivian Shimanuki, Michelyn Palmer and Pat McGarvey for data tabulation and preparation of the manuscript.

\section{REFERENCES}

Baird, G. D., Lomax, M. A., Symonds, H. W. \& Shaw, S. R. (1980). Net hepatic and splanchnic metabolism of lactate, pyruvate and propionate in dairy cows in vivo in relation to lactation and nutrient supply. Biochemical Journal 186, 47-57.

Bergman, E. N. \& Heitmann, R. N. (1978). Metabolism of amino acids by the gut, liver, kidneys, and periphera! tissues. Federation Proceedings 37, $1228-1232$.

Briggs, S. \& Freedland, R. A. (1976). Effect of ornithine and lactate on urea synthesis in isolated hepatocytes. Biochemical Journal 160, 205-209.

Demaugre, F., Buc, H., Girard, J. \& Leroux, J. (1982). Role of the mitochondrial metabolism of pyruvate on the regulation of ketogenesis in rat hepatocytes. Metabolism 32, 4048

Diamond, M. P., Rollings, R. C., Steiner, K. E., Williams, P. E., Lacy, W. W. \& Cherrington, A. D. (1988). Effect of alanine concentration independent of changes in insulin and glucagon on alanine and glucose homeostasis in the conscious dog. Metabolism 37, 28-33.

Genuth, S. M. (1973). Effect of oral administration of alanine in fasting obese subjects. Metabolism 22, 927-934.

Hetenyi, G., Layberry, R. A., Foster, D. M. \& Berman, M. (1980). Transfer of carbon atoms among circulating glucose, alanine, and lactate in pancreatectomized dogs. American Journal of Physiology 239, E39-E44.

Krebs, H. A., Lund, P. \& Stubbs, M. (1979). Interrelation between gluconeogenesis and urea synthesis. In Gluconeogenesis: Its Regulation in Mammalian Species, pp. 269-291 [R. Hanson and M. A. Mehlan, editors]. New York: Wiley.

Lomax, M. A. \& Baird, G. D. (1983). Blood flow and nutrient exchange across the liver and gut of the dairy cow. British Journal of Nutrition 49, 481-496.

Newsholme, E. A. \& Leach, A. R. (1983). Amino acid metabolism. In Biochemistry for the Medical Sciences, pp. 382 440. New York: Wiley.

Nosandi, E., Datta, H., Hodson, A. \& Alberti, K. G. M. M. (1980), A possible mechanism for the anti-ketogenic action of alanine in the rat. Biochemical Journal 190, 323-332.

Owens, F. N., Zinn, R. A.\& Kim, Y. K. (1986). Limits to starch digestion in the ruminant small intestine. Journal of Animal Science 63, 1634-1648.

Ozand, P. T., Reed, W. D., Girard, J., Hawkins, R. L., Collins, R. M., Tildon, J. T. \& Cornblath, M. (1977). Hypoketonaemic effect of $L$-alanine. Specific decrease in blood concentrations of 3-hydroxybutyrate in the rat. Biochemical Journal 164, 557-564.

Ozand, P. T., Reed, W. D., Hawkins, R. L., Stevenson, J. H., Tildon, J. T. \& Cornblath, M. (1978). Effect of $\mathrm{L}$-alanine infusion on gluconeogenesis and ketogenesis in the rat in vivo. Biochemical Journal 170, 583-591.

Reynolds, C. K., Huntington, G. B., Tyrrell, H. F. \& Reynolds, P. J. (1988a). Net portal-drained visceral and hepatic metabolism of glucose, L-lactate and nitrogenous compounds in lactating Holstein cows. Journal of Dairy Science 71, 1803-1812.

Reynolds, C. K., Huntington, G. B., Tyrrell, H. F. \& Reynolds, P. J. (1988b). Net metabolism of volatile fatty 
acids, B-OH-hydroxybutyrate, nonesterified fatty acids, and blood gasses by portal-drained viscera and liver of lactating Holstein cows. Journal of Diary Science 71, 2395-2405.

Reynolds, C. K., Lapierre, H., Tyrrell, H. F., Elsasser, T. H., Gaudreau, P. \& Brazeau, P. (1990). Growth hormone-releasing factor (GRF) and intake affect net visceral tissue metabolism in growing beef steers. Journal of Animal Science 67, Suppl. 1, 534.

Reynolds, C. K., Tyrrell, H. F. \& Reynolds, P. J. (1991). Effects of forage-to-concentrate ratio and intake on energy metabolism in growing beef heifers: net nutrient metabolism by visceral tissues. Journal of Nutrition 121, $1004-1015$.

Shepherd, A. P. (1982). Metabolic control of intestinal oxygenation and blood flow. Federation Proceedings 41, 2084-2089.

Statistical Analysis System (1988). Statistical Analysis System User's Guide. Cary, NC: SAS Institute.

Williamson, D. H. (1974). L-Alanine determination with alanine dehydrogenase. In Methods of Enzymatic Analysis, pp. 1679-1682 [H. A. Bergmeyer, editor]. New York: Academic Press.

Wolff, J. E. \& Bergman, E. N. (1972). Gluconeogenesis from plasma amino acids in fed sheep. American Journal of Physiology 223, 455-460. 